\title{
Combination of rapamycin and garlic-derived S-allylmercaptocysteine induces colon cancer cell apoptosis and suppresses tumor growth in xenograft nude mice through autophagy/p62/Nrf2 pathway
}

\author{
SIYING LI ${ }^{1}$, GUANG YANG $^{2}$, XIAOSONG ZHU ${ }^{1}$, LIN CHENG $^{3}$, YUEYUE SUN $^{1}$ and ZHONGXI ZHAO ${ }^{1}$ \\ ${ }^{1}$ School of Pharmaceutical Sciences, Shandong University, Jinan, Shandong 250012; ${ }^{2}$ Department of Joint Surgery, \\ Shandong Provincial Hospital Affiliated to Shandong University, Jinan, Shandong 250021; \\ ${ }^{3}$ Department of Endocrinology, Heze Municipal Hospital, Heze, Shandong 274000, P.R. China
}

Received February 9, 2017; Accepted July 10, 2017

DOI: $10.3892 /$ or.2017.5849

\begin{abstract}
The natural plant-derived product S-allylmercaptocysteine (SAMC) has been studied in cancer therapy as a single and combination chemotherapeutic agent. The present study was employed to verify the combination use of SAMC and rapamycin that is the mTOR inhibitor with anticancer ability but has limited efficacy due to drug resistance, and to explore the underlying mechanisms. We combined rapamycin and SAMC for colorectal cancer treatment in the HCT-116 cancer cells and a xenograft murine model. The in vivo study was established by xenografting HCT-116 cells in BALB/c nude mice. It was found that the combination therapy had enhanced tumor-suppressing ability with the upregulation of the $\mathrm{Bax} / \mathrm{Bcl}-2$ ratio as a consequence of activated apoptosis, inhibition of autophagic activity and prevention of Akt phosphorylation. The rapamycin and SAMC combination activated antioxidant transcription expressions of Nrf2 and downstream gene NQO1. Concomitantly, autophagosome cargo p62 was downregulated, indicating that the p62 played a negative-regulatory role between $\mathrm{Nrf} 2$ and autophagy. Our results show that the combination of SAMC and rapamycin enhanced the anticancer ability, which could be used for the treatment of colorectal cancer. The underling mechanism of autophagy/p62/Nrf2 pathway discovered may provide
\end{abstract}

Correspondence to: Professor Zhongxi Zhao, School of Pharmaceutical Sciences, Shandong University, 44 West Wenhua Road, Jinan, Shandong 250012, P.R. China

E-mail: zxzhao@sdu.edu.cn

Abbreviations: SAMC, S-allylmercaptocysteine; mTOR, mammalian target of rapamycin; OSCs, organosulfur compounds; Nrf2, nuclear factor (erythroid-derived 2)-like 2; HO-1, heme oxyegenase-1; NQO1, $\mathrm{NAD}(\mathrm{P}) \mathrm{H}$ : quinone oxidoreductase 1; TCM, traditional Chinese medicine

Key words: rapamycin, S-allylmercaptocysteine, autophagy, Nrf2, colorectal cancer, p62 a new direction for drug development, especially for traditional Chinese medicines.

\section{Introduction}

Globally, colorectal cancer (CRC) is the second and third most common causes of cancer in women ( $9.2 \%$ of diagnoses) and men $(10.0 \%)$, respectively (1). Colorectal cancer death ranks fourth after lung, stomach, and liver cancer, which is more common in developed countries (2). Treatments used for colorectal cancer include rational combination of surgery, radiation therapy, chemotherapy and targeted therapy.

In recent years, autophagy has become a topic of debate in cancer (3). Autophagy is a conserved lysosomal degradation process of intracellular recycling and degraded metabolites, and can maintain the cellular homeostasis and adaption to stress conditions. In cancer, autophagy plays a complex role in tumor initiation and growth. It can either induce cancer cell death by eliminating carcinogenic factors and damaged organelles in the initial stage of cancer; or, promote cancer cell survival by intracellular recycling of degraded metabolites to cope with starvation or stress conditions in cancer progression (4). In addition, autophagy may contribute to drug resistance due to an adaptive response to chemotherapy and radiation therapy (5).

The mammalian target of rapamycin (mTOR) signaling plays a role in suppressing autophagy (6). The dysregulation of the mTOR activity is found linking to the initiation and development of many human tumors (7). The most established mTOR inhibitors such as rapamycin have been evaluated as anticancer agents, and used in the treatment of cancer in clinical trials (8).

Rapamycin inhibits mTOR by binding the FK506 binding protein 1A, $12 \mathrm{kDa}$ (FKBP-12) (9). However, the use of mTOR inhibitors is limited because of the occurrence of cancer escape due to drug resistance. The mechanism is related to oncogene Akt reactivation by negative mTOR-PI3K feedback (10).

The use of natural plant-derived products in cancer therapy has gained attention in recent years. Garlic, a plant within the 
genus Allium, has been explored by focusing on many organosulfur compounds (OSCs). S-allylmercaptocysteine (SAMC), the water-soluble fraction, is one of the major part in OSCs (11). SAMC has been studied in various cancer cells for its antiproliferative effect $(12,13)$, and in vivo studies also showed tumor suppressive ability $(14,15)$. Moreover, SAMC is a good adjuvant in combination with chemopreventive agents (16).

The relationship between autophagy and Nrf2 pathway has been studied, promoting a series of antioxidant programs (17). Also, garlic and garlic extract has shown to facilitate Nrf2-HO-1 signaling in endothelial (18) and B35 neural cells (19). Nuclear factor (erythroid-derived 2)-like 2, also known as $\mathrm{Nrf} 2$, is a transcription factor that protects against oxidative damage triggered by injury and inflammation. Nrf2 is a basic leucine zipper (bZIP) protein that regulates a variety of detoxification enzymes such as heme oxyegenase-1 (HO-1), NAD(P)H: quinone oxidoreductase 1 (NQO1). In many cancer cell lines and tumor tissues, high level of Nrf2 is detected, and Nrf2 is thought to play dual roles in cancer cell growth and survival (20).

In this study, we used both rapamycin and SAMC as anticancer reagents in human colon cancer cells and tumor xenograft mice, and investigated the underlying mechanisms of tumor growth inhibition, especially the relationship between autophagy and Nrf2.

\section{Materials and methods}

Reagents. SAMC (purity of 99\%) was synthesized and purified in our laboratory with a modified procedure as previously reported (21). SAMC was freshly prepared as a stock solution in PBS for the in vitro assay and was suspended in $10 \%(\mathrm{w} / \mathrm{v})$ L-dextrose, $1 \%$ (w/v) gum Arabic (Sigma-Aldrich, St. Louis, MO, USA) for application in mice.

Cell culture. The human colorectal cancer cell line HCT-116 was purchased from American Type Culture Collection (ATCC, Manassas, VA, USA). HCT-116 cells were cultured in RPMI-1640 (Hyclone, Logan, UT, USA) supplemented with $10 \%$ fetal bovine serum (BI, Cromwell, CT, USA), $100 \mathrm{U} / \mathrm{ml}$ of penicillin and $100 \mathrm{mg} / \mathrm{ml}$ streptomycin (Solarbio, Beijing, China). All cells were maintained in a humidified incubator at $37^{\circ} \mathrm{C}$ in $5 \% \mathrm{CO}_{2} / 95 \%$ air.

Cell viability assay. A stock solution of SAMC (5 mM) was prepared fresh in PBS. Rapamycin was dissolved in dimethyl sulfoxide (DMSO) as a 5-mM stock solution and dilutions were made in RPMI-1640. The total DMSO concentrations were kept below $0.05 \%(\mathrm{v} / \mathrm{v})$, which showed no influence on cell growth. Cells were seeded in 96-well plates at a concentration of $1.5 \times 10^{3}$ cells/well. After $24 \mathrm{~h}$, cells were categorized into five groups: control, rapamycin (Rapa) $(0.5 \mu \mathrm{M})$, SAMC (200 and $400 \mu \mathrm{M})$ and Rapa+SAMC combination group. The two doses of SAMC were selected based on our previous experiment (data not shown). Cell viability was measured by the SRB method. Briefly, the treated cells were then fixed with $10 \%$ TCA for $1 \mathrm{~h}$ at $4^{\circ} \mathrm{C}$, the 96 -well plates were washed three times with distilled water and allowed to dry in the air. Each well was added with $100 \mu \mathrm{l}$ of sulphorhodamine (SRB) solution and the staining was completed at room temperature for $15 \mathrm{~min}$. The SRB stain solution was removed by washing the plates quickly with $1 \%(\mathrm{v} / \mathrm{v})$ acetic acid three times, and the plates were dried in the air. The dried materials in each well were solubilized by adding $200 \mu \mathrm{l}$ of $10 \mathrm{mM}$ unbuffered Tris base ( $\mathrm{pH} \mathrm{10.5).}$ The cell viability was detected by measuring the absorbance at $540 \mathrm{~nm}$ on a plate reader (Safire2, Tecan, France). All experiments were repeated at least three times.

DAPI staining. The HCT-116 cells were seeded into 24-well plates for $24 \mathrm{~h}$. Then Rapa and SAMC (200 and $400 \mu \mathrm{M})$ were directly added to the well and incubated for $48 \mathrm{~h}$. The treated cells were washed with PBS and fixed with cold methanol/ acetone $\left(1: 1\right.$, stored at $\left.-20^{\circ} \mathrm{C}\right)$ for $5 \mathrm{~min}$ at room temperature. The solution was removed and washed with PBS, and then incubated with the DAPI solution for $10 \mathrm{~min}$ at room temperature. Fluorescent cells were observed under a fluorescence microscope (Olympus, Tokyo, Japan).

Animal experiments. Female BALB/c nude mice (16 g, aged 5-6 weeks) were purchased from Institute of Laboratory Animal Sciences, Cams\&Pumc (Beijing, China, SCXK 2014-0004). Mice were housed under standard conditions $(12: 12 \mathrm{~h}$ light/dark cycle at $25 \pm 2{ }^{\circ} \mathrm{C}$ and $40-70 \%$ humidity) in specific pathogen-free (SPF) conditions, with ad libitum access to food and water. The protocol of the animal experiments were performed in accordance with the institutional guidelines of the Animal Care and Use Committee of Shandong University.

In vivo xenograft implantation and tumor growth. The human colorectal adenocarcinoma HCT-116 cells used for implantation were harvested during log phase growth and resuspended in phosphate-buffered saline (PBS) at $5 \times 10^{7}$ cells $/ \mathrm{ml}$. Each $\mathrm{Balb} / \mathrm{c}$ nu/nu mouse was injected s.c. in the right flank with $1 \times 10^{7}$ cells $(0.2 \mathrm{ml}$ cell suspension). When tumor volume reached $\sim 100 \mathrm{~mm}^{3}$, mice were randomly divided into four groups $(\mathrm{n}=6)$ : the control, rapamycin (Rapa) $(5 \mathrm{mg} / \mathrm{kg} / \mathrm{per}$ two days), S-allylmercaptocysteine (SAMC) $(300 \mathrm{mg} / \mathrm{kg} / \mathrm{d})$, and Rapa + SAMC combination. All groups were treated for 28 days, Rapa was given as intraperitoneal injection three times a week, and SAMC was given by oral gavage daily.

Mice were carefully observed daily, and tumor volumes and body weights were measured every four days. The diameters of the tumors were measured with a caliper, and the tumor volume was calculated using the formula: $\mathrm{V}=1 / 2 \times \mathrm{L} \mathrm{x} \mathrm{W}^{2}$, where length $(\mathrm{L})$ and width $(\mathrm{W})$ were determined in millimeters $(\mathrm{mm})$. The inhibition rate $(\%)$ of tumor growth was defined as the ratio of tumor weight to that of the control.

At the end of the experiment, all animals were euthanized. The tumor weight and spleen, liver, kidney weight were measured. Tumors were resected and snap-frozen in liquid nitrogen or transferred at $-80^{\circ} \mathrm{C}$ for western blotting and PCR.

Western blot analysis. Tumor tissues were homogenized with RIPA lysis buffer [50 mM Tris ( $\mathrm{pH} 7.4), 150 \mathrm{mM} \mathrm{NaCl}$, $1 \%$ Triton $\mathrm{X}-100,1 \%$ sodium deoxycholate, $0.1 \%$ SDS] containing protease and phosphatase inhibitor cocktails (Roche), at $4^{\circ} \mathrm{C}$ with vortexing. The total protein concentration was determined by the BCA protein assay reagent (Pierce Biomedical Co., Rockford, IL, USA). Tissue lysates were separated using 12\% SDS-PAGE and electro-transferred onto the polyvinylidene difluoride (PVDF) membrane. The 
Table I. Effect of rapamycin and SAMC on the tumor growth of HCT-116 xenografts in mice.

\begin{tabular}{|c|c|c|c|c|}
\hline \multirow[b]{2}{*}{ Group } & \multicolumn{2}{|c|}{ Tumor volume $\left(\mathrm{mm}^{3}\right)$} & \multirow[b]{2}{*}{ Average tumor weight (g) } & \multirow[b]{2}{*}{ Inhibition rate $(\%)$} \\
\hline & Beginning & At the end & & \\
\hline Control & $130.93 \pm 25.34$ & $1,242.72 \pm 311.60$ & $2.42 \pm 0.59$ & \\
\hline Rapa & $135.22 \pm 22.24$ & $390.97 \pm 62.71^{\mathrm{a}}$ & $0.88 \pm 0.17^{\mathrm{a}}$ & 63.64 \\
\hline SAMC & $110.47 \pm 17.64$ & $603.42 \pm 157.42^{\mathrm{a}, \mathrm{b}}$ & $0.99 \pm 0.14^{\mathrm{a}}$ & 59.09 \\
\hline Rapa+SAMC & $129.79 \pm 20.92$ & $307.84 \pm 53.36^{\mathrm{a}, \mathrm{b}}$ & $0.48 \pm 0.11^{\mathrm{a}, \mathrm{b}}$ & 80.17 \\
\hline
\end{tabular}

Mean $\pm \mathrm{SD}, \mathrm{n}=6 .{ }^{\mathrm{a}} \mathrm{p}<0.05$ vs control. ${ }^{\mathrm{b}} \mathrm{p}<0.05$ vs Rapa.

protein expression levels were determined using primary antibodies with the appropriate dilution. The PVDF membranes were washed in Tris-buffered saline (TBS) containing $0.1 \%$ Tween-20 (TBST) and incubated with appropriate secondary antibodies. The immunoreactive bands were visualized by an enhanced chemiluminescence reagent (Millipore) using Alphalmager HP system (Cell Biosciences, USA).

The density of each band was measured using Image-Pro Plus, standardized by the density of $\beta$-actin. The primary antibodies included those of anti-LC3 (L7543, Sigma-Aldrich), anti-p62 (ab91526, Abcam), anti-Bcl-2 (ab32124, Abcam), anti-Bax (ab32503, Abcam), anti-Nrf2 (C-20) (sc-722, Santa Cruz Biotechnology), anti-NQO1 (ab34173, Abcam), anti-Akt (sc-8312, Santa Cruz Biotechnology), anti-phospho-Akt/Sre-473 (sc-135651, Santa Cruz Biotechnology), p53(ab28, Abcam) and $\beta$-actin (TA-09, ZSGB-BIO).

Quantitative real-time ( $q$-PCR). Total RNA from the tumor was extracted using TRIzol Reagent (Invitrogen Corp.). Reverse transcription reactions (SuperScript III First-Strand Synthesis system for RT-PCR; Invitrogen Corp., Carlsbad, CA, USA) were performed with $0.5 \mu \mathrm{g}$ of DNase I (Qiagen)-treated RNA. PCR and quantitative real-time PCR (q-PCR) were carried out using the CFX96 Touch $^{\mathrm{TM}}$ Real-Time PCR Detection system (Bio-Rad, Laboratories, Inc.). Expression levels of target genes were normalized by concurrent measurement of glyceraldehyde-3-phosphate dehydrogenase (GAPDH) mRNA levels. Primers for target gene amplification were as follows: Nrf2 (NM_010902.3): 5'-ATGATGGACTTGGA GTTGCC-3', 5'-TCCTGTTCCTTCTGGAGTTG-3'; NQO1 (NM_008706.5):5'-CGGTATTACGATCCTCCCTCAACA-3, 5'-AGCCTCTACAGCAGCCTCCTTCAT-3'; GAPDH (NM_008084.2):5'-ATGTTCCAGTATGACTCCACTCACG-3', 5'-GAAGACACCAGTAGACTCCACGACA-3'.

Measurements of the oxidative stress markers and antioxidant enzyme activities. A $10 \%$ liver tissue homogenate was prepared with the phosphate buffer saline $(50 \mathrm{mM}, \mathrm{pH} 7.4)$ using Teflon homogenizer. A part of the homogenate was mixed with an equal volume of $10 \%$ trichloroacetic acid (TCA) and centrifuged at 3,000 rpm for $15 \mathrm{~min}$ at $4^{\circ} \mathrm{C}$. The supernatant was used to determine the content of glutathione (GSH-Px) and malondialdehyde (MDA) (oxidative stress marker) enzymes. The remaining part of the homogenate was centrifuged at $12,000 \mathrm{~g}$ for $45 \mathrm{~min}$ at $4^{\circ} \mathrm{C}$ and the supernatant was used for estimation of antioxidant enzymes activities as superoxide dismutase (SOD) and catalase (CAT) enzymes. All treatments were conducted in an ice bath. The activities of GSH-Px, MDA, SOD and CAT were then assayed using commercial reagent kits (Nanjing Jiancheng Bioengineering Institute, Nanjing, China) according to the manufacturer's instructions.

Statistical analysis. Data are shown as mean \pm SD. Statistical differences were analyzed by one-way ANOVA followed by Bonferroni test for multiple comparisons using GraphPad Prism software. Differences were considered significant at $\mathrm{p}<0.05$. Statistical analysis was performed with SPSS/ Win 13.0 software (SPSS, Inc., Chicago, IL, USA).

\section{Results}

Rapamycin and SAMC combination inhibits colon cancer cell proliferation and induces apoptosis in HCT-116 cells. To assess the antiproliferative activity of rapamycin, SAMC and co-treatment group, HCT-116 cells were exposed to different concentrations (200 and $400 \mu \mathrm{M})$ of SAMC for $48 \mathrm{~h}$ and assayed for proliferation using the SRB method. As shown in Fig. 1A, the proliferation of HCT-116 cells was significantly suppressed by Rapa, SAMC, and the combination.

SAMC has been shown to induce colorectal carcinoma cell apoptosis (22). To detect the cell apoptosis induced by rapamycin and SAMC, the HCT-116 cells were stained with DAPI to assess the morphological change in this study. The cells exhibited typical morphological signs of apoptosis, such as fragmented nuclei and apoptotic bodies, as evidenced by the arrows in Fig. 1B.

SAMC potentiates antitumor effect of rapamycin in mouse subcutaneous HCT-116 xenograft tumor models. The antitumor effect of rapamycin and SAMC was evaluated in the mouse model of HCT-116 xenografts. Compared with the control group, Rapa treatment $(10 \mathrm{mg} / \mathrm{kg}$ per day three times a week) significantly inhibited tumor growth (Fig. 2A and B) by $63.64 \%(\mathrm{p}<0.05)$, while SAMC alone inhibited tumor growth by $59.09 \%(\mathrm{p}<0.05)$, respectively. However, the tumor growth inhibition rate of the combination reached $80.17 \%(\mathrm{p}<0.05)$ compared with the control. The average tumor volumes on day 28 were $390.97 \pm 62.71,603.42 \pm 157.42$ and $307.84 \pm 53.36 \mathrm{~mm}^{3}$ in Rapa, SAMC and combination groups, respectively, compared to $1,242.72 \pm 311.60 \mathrm{~mm}^{3}$ of control group (Table I). The body weight of mice decreased in the control group, and Rapa treatment showed a similar 
A
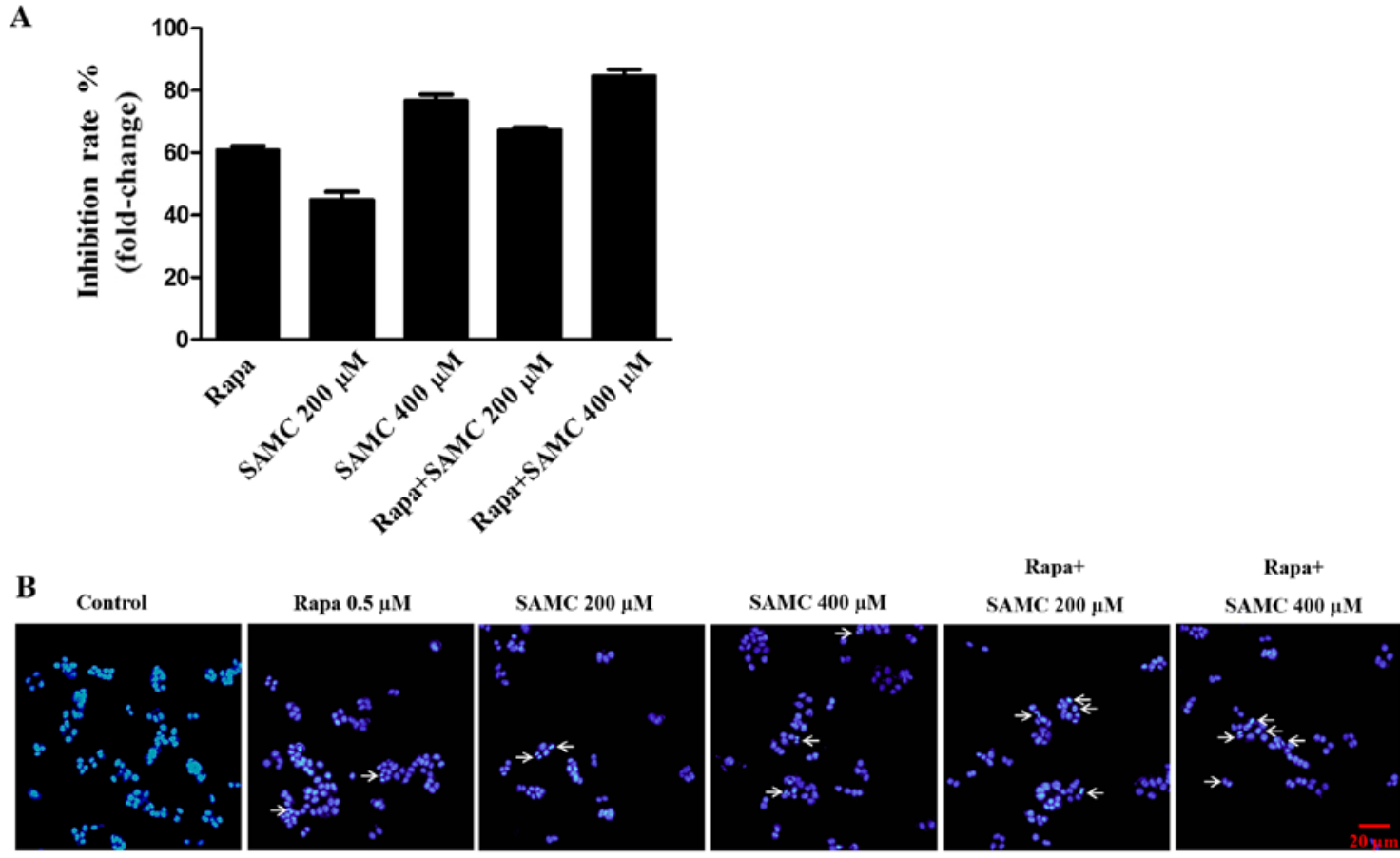

Figure 1. Rapamycin and SAMC inhibit HCT-116 cell growth and induce cell apoptosis. (A) The inhibition effect of Rapa (0.5 $\mu \mathrm{M})$ and SAMC (200 and $400 \mu \mathrm{M}$ ) on HCT-116 cell growth was estimated by the SRB assay. Cells were exposed to Rapa and SAMC for $48 \mathrm{~h}$, followed by SRB assay. (B) Rapa and SAMC induced apoptosis showed by DAPI staining of HCT-116 cells after 48-h treatment. Data represent the mean \pm SD of three independent experiments.

A

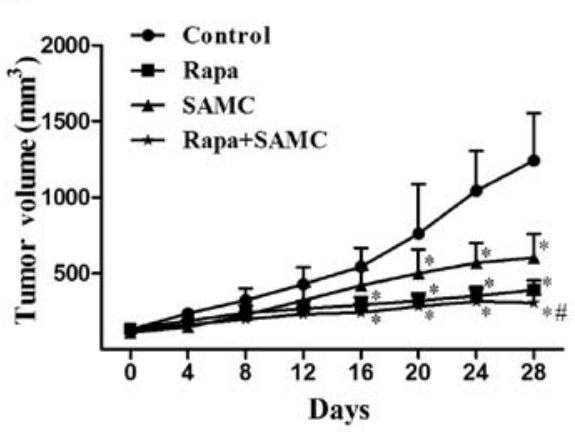

C

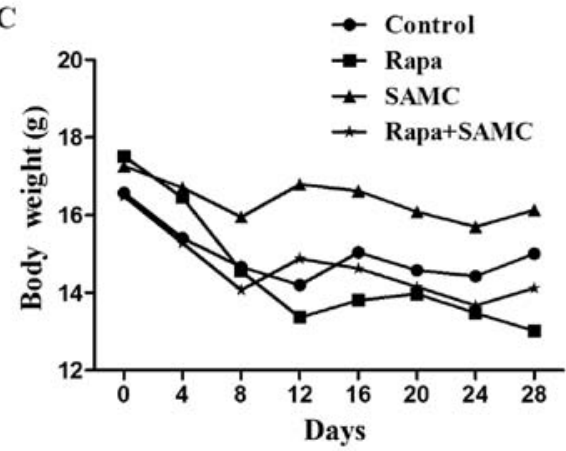

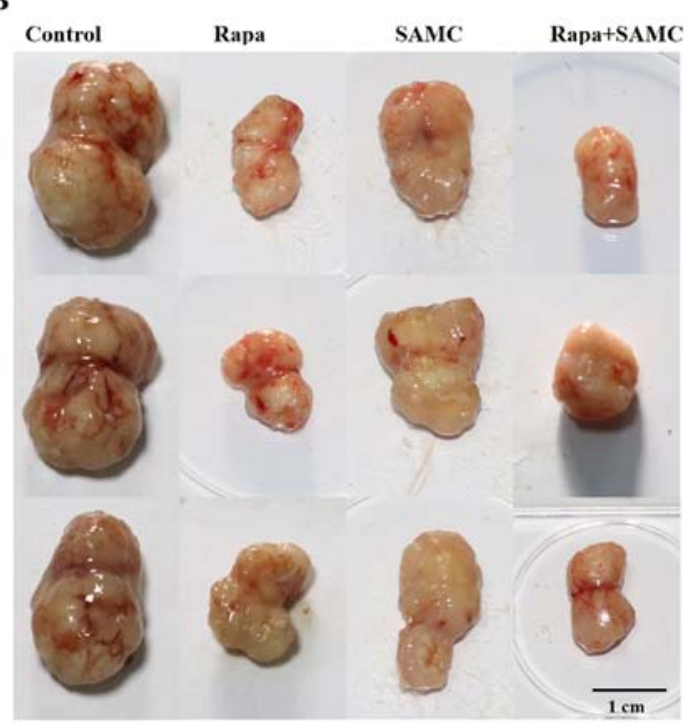

Figure 2. The combination of rapamycin and SAMC inhibit tumor growth in nude mice bearing HCT-116 xenograft. (A) Mice were administered Rapa $(5 \mathrm{mg} / \mathrm{kg}$, i.p., three times per week) and SAMC $(300 \mathrm{mg} / \mathrm{kg}$, o.p., daily) for 28 days, and tumor volume were measured every other four days (n=6). At the end of treatment, Rapa and SAMC exhibited significant tumor growth inhibition effect, and this effect was strengthened when combination use. Bars, SD. (B) Representative images of tumor tissues in each group. (C) The body weight change of mice in each group. " $\mathrm{p}<0.05$ vs control; " $\mathrm{p}<0.05$ vs Rapa.

degree of body weight loss to the control; but SAMC treatment showed no obvious weight loss. Moreover, SAMC and Rapa combination partially reversed the weight loss caused by Rapa (Fig. 2C).
Rapamycin and SAMC combination regulates autophagy process and induces apoptosis. In order to examine whether autophagy inhibition can enhance the antitumor ability of rapamycin on colorectal cancer, we tested the tumor tissues of 

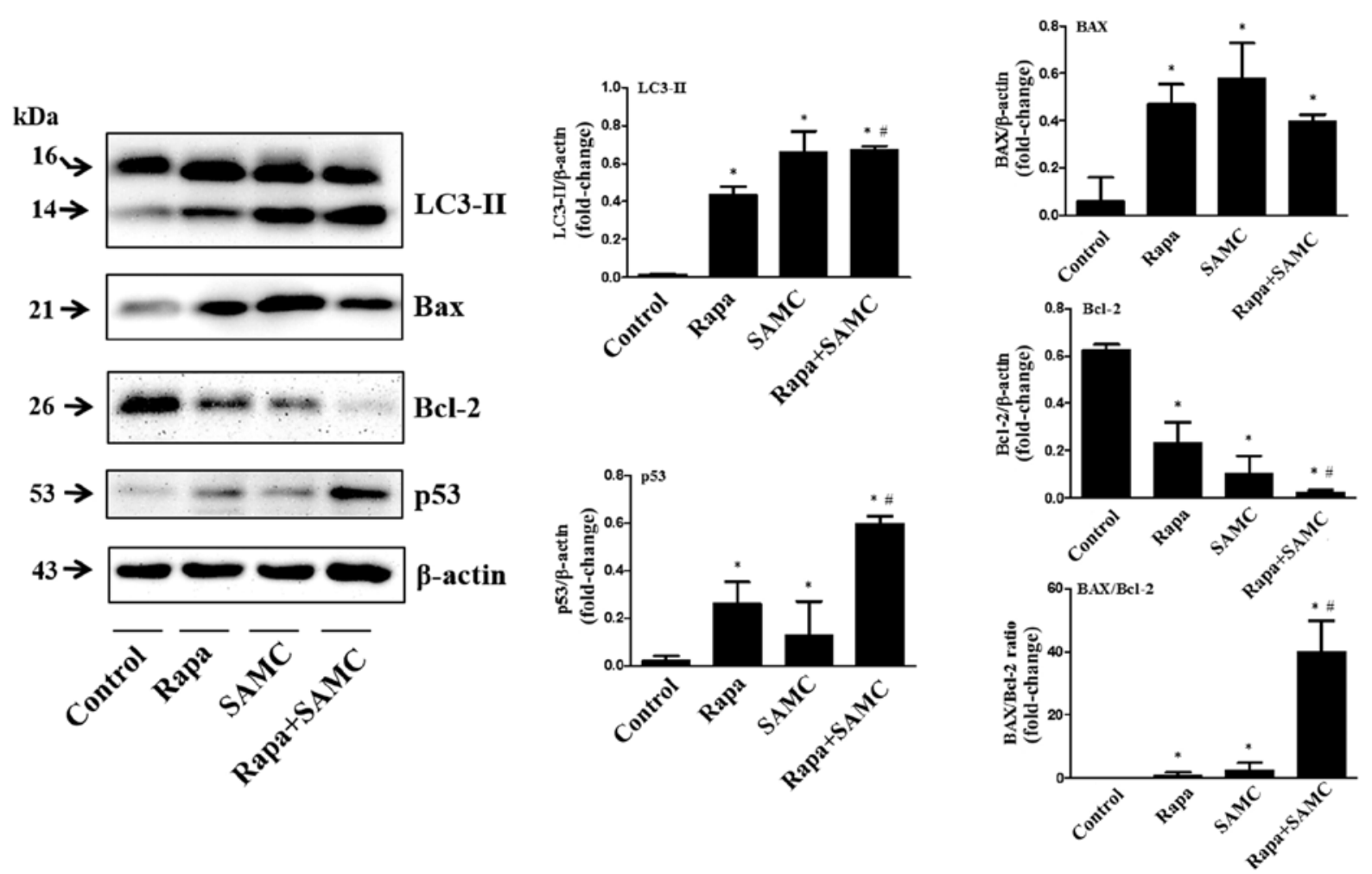

Figure 3. The effect of rapamycin and SAMC treatment on autophagy and apoptosis. The xenografts tumor tissue were examined by western blotting ( $\mathrm{n}=4$ in each group). Protein expression levels were normalized to actin. The autophagy marker LC3-II was upregulated by Rapa treatment and enhanced in Rapa and SAMC combination group. The Rapa and SAMC treatment can induce tumor cell apoptosis, which was p53-dependent, as the increased Bax/Bcl-2 ratio and p53 expression. Data represent the mean \pm SD of three independent experiments (bars, SD). ${ }^{*} \mathrm{p}<0.05$ vs control; ${ }^{\#} \mathrm{p}<0.05$ vs Rapa.

HCT-116 xenografts treated with both Rapa and SAMC. The autophagy-related protein, LC3-II, represent the autophagic activity (23). Western blot analysis showed that the LC3-II expression level was increased by Rapa alone and further increased when combined with SAMC (Fig. 3).

Apoptotic protein Bax and Bcl-2 expression levels were examined in Rapa with/without SAMC-treated tumors. Rapa and SAMC increased the Bax expression, while downregulated the Bcl-2 expression. The Bax/Bcl-2 ratio increased $\sim 40$-fold in the Rapa and SAMC combination group compared to the control (Fig. 3). Tumor suppressor p53 plays a critical role in the induction of apoptosis, which was also examined by western blotting in this study. Rapa increased p53 expression, and further enhanced in the combination group (Fig. 3), indicating that the apoptosis may be p53-dependent.

Rapamycin and SAMC combination reverses rapamycininduced upregulation of Akt signaling. The serine/threonine kinase mTOR is a downstream effector of the PI3K/AKT pathway, and mTOR inhibition consequently reactivates Akt signaling (24). The Akt signaling activation plays a role of drug-resistance in rapamycin-related cancer therapy, so we examined the Akt phosphorylation in tumor tissues. Rapa treatment alone increased Akt phosphorylation, but SAMC alone did not change the Akt status. However, the Akt activation was inhibited in the combination group (Fig. 4).

Co-treatment of rapamycin and SAMC suppresses tumor growth by activating antioxidant system via regulating $p 62$. To further explore the mechanism of tumor-suppressing effects

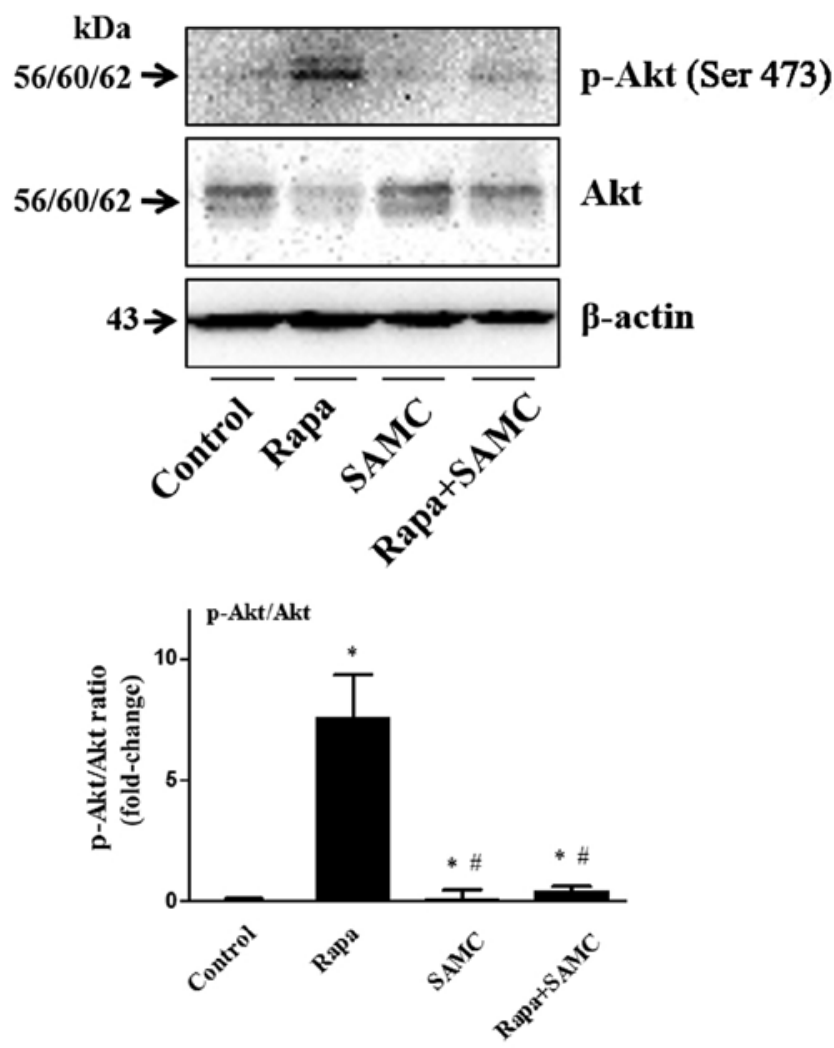

Figure 4. The combination of Rapa with SAMC decreased rapamycininduced Akt activation. Western blotting results showed that Rapa treatment increased Akt phosphorylation (p-Akt) expression, but was reversed by SAMC combination treatment. Data represent the mean \pm SD of three independent experiments (bars, SD). ${ }^{*} \mathrm{p}<0.05$ vs control; ${ }^{\#} \mathrm{p}<0.05$ vs Rapa. 

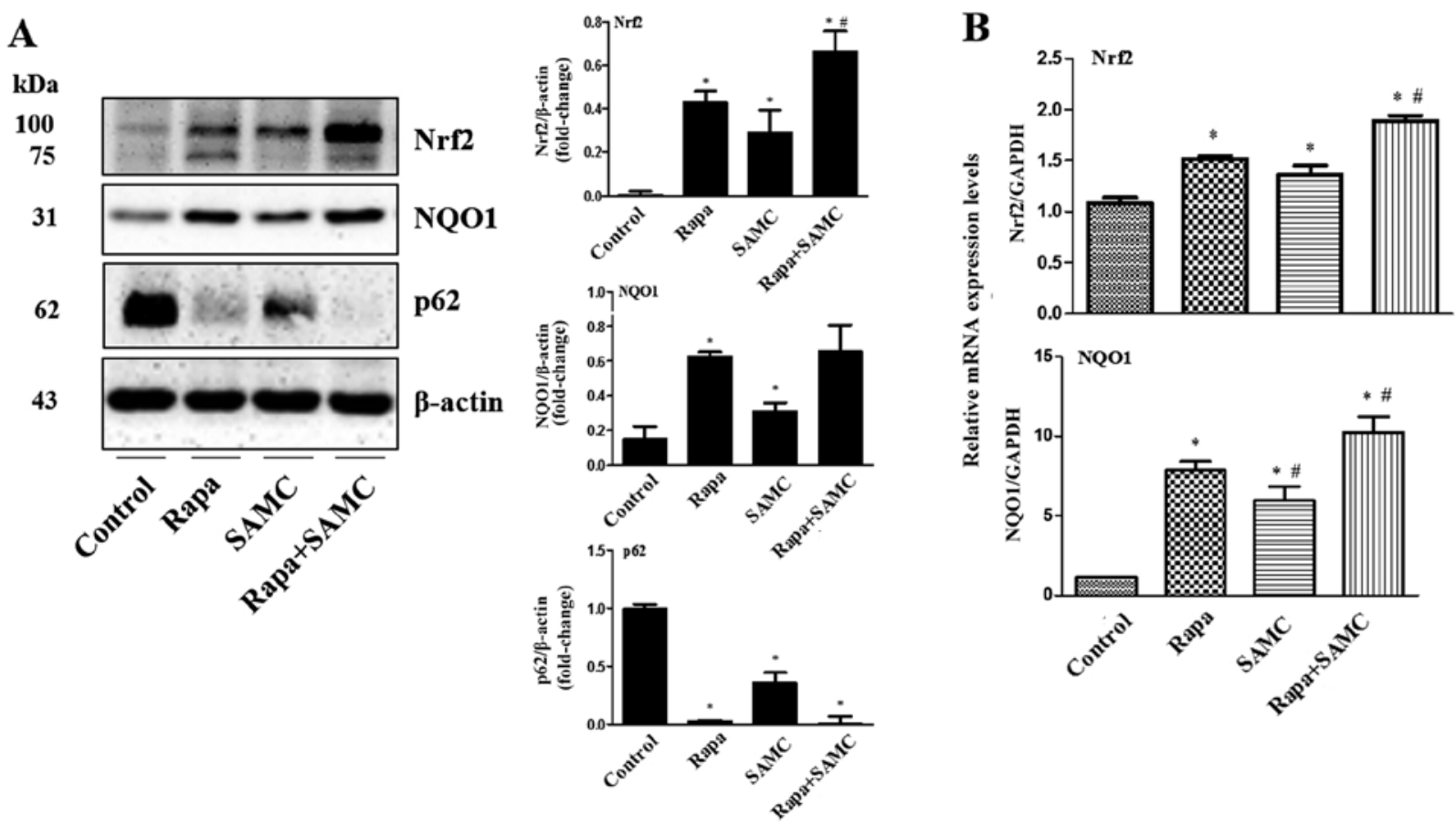

Figure 5. Rapamycin and SAMC activate the antioxidant system in tumor tissue. The antioxidative stress transcriptor Nrf2 and its downstream gene NQO1 expression were increased by western blot analysis (A) and q-PCR (B) in Rapa and SAMC co-treatment ( $\mathrm{n}=4$ in each group). The p62 expression was also examined by western blotting (A), which showed a negative expression compare with Nrf2 and NQO1. Data represent the mean \pm SD of three independent experiments (bars, SD). ${ }^{*} \mathrm{p}<0.05$ vs control; $" \mathrm{p}<0.05$ vs Rapa.

Table II. SOD, GSH-Px, CAT activity and MDA level in HCT-116 xenograft mouse liver tissue.

\begin{tabular}{llccc}
\hline Group & \multicolumn{1}{c}{$\begin{array}{c}\text { SOD } \\
\text { (U/mg protein) }\end{array}$} & $\begin{array}{c}\text { GSH-Px } \\
\text { (U/mg protein) }\end{array}$ & $\begin{array}{c}\text { CAT } \\
\text { (U/mg protein) }\end{array}$ & $\begin{array}{c}\text { MDA } \\
\text { (nmol/mg protein) }\end{array}$ \\
\hline Control & $167.42 \pm 11.26$ & $103.40 \pm 12.33$ & $11.08 \pm 0.33$ & $5.76 \pm 0.18$ \\
Rapa & $241.04 \pm 12.27^{\mathrm{a}}$ & $164.21 \pm 10.38^{\mathrm{a}}$ & $16.20 \pm 0.38^{\mathrm{a}}$ & $3.05 \pm 0.06^{\mathrm{a}}$ \\
SAMC & $216.44 \pm 9.43^{\mathrm{a}}$ & $136.74 \pm 20.05$ & $13.74 \pm 0.05^{\mathrm{a}}$ & $3.28 \pm 0.13^{\mathrm{a}}$ \\
Rapa+SAMC & $287.19 \pm 4.53^{\mathrm{a}, \mathrm{b}}$ & $183.74 \pm 22.05^{\mathrm{a}}$ & $18.30 \pm 2.67^{\mathrm{a}}$ & $2.81 \pm 0.04^{\mathrm{a}, \mathrm{b}}$ \\
\hline
\end{tabular}

Mean $\pm \mathrm{SD}, \mathrm{n}=6 .{ }^{\mathrm{a}} \mathrm{p}<0.05$ vs control. ${ }^{\mathrm{b}} \mathrm{p}<0.05$ vs Rapa.

by the combination of rapamycin and SAMC, we examined the antioxidant transcription factor Nrf2 and its downstream gene expression such as NQO1. In Fig. 5, western blotting and q-PCR results show that the Nrf2 expression was elevated in the Rapa-treated group compared to the control, and further enhanced when combined with SAMC. While the downstream gene NQO1 expression was consistent with Nrf2 expression.

Autophagy has been shown to suppress tumorigenesis and the key associate point relied on p62/SQSTM, promoting oxidative stress status (25). Our results indicate that the Rapa, SAMC and combination groups downregulated p62 level compared to the control (Fig. 5A).

Effects of rapamycin and SAMC on SOD, GSH-Px, CAT and $M D A$. To further explore the mechanisms of rapamycin, SAMC and combination for tumor growth inhibition, we determined the activities of antioxidant enzyme SOD, GSH-Px and CAT as well as the lipid peroxidation marker MDA content in mouse liver tissues. The Rapa alone and combination treatment decreased oxidative stress as indicated by enhanced SOD, GSH-Px and CAT contents and diminishment of MDA level in liver tissues (Table II). The levels of SOD, GSH-Px and CAT were increased by $43.97,58.81$ and $11.08 \%$, respectively, and the MDA was decreased by $47.05 \%$ in the Rapa-treated mice compared to the control group. The antioxidant effect of the combination treatment was further strengthened, the SOD, GSH-Px and CAT values were $287.19 \pm 4.53,183.74 \pm 22.05$ and $18.30 \pm 2.67$ (U/mg protein), respectively, and MDA content was diminished to $2.81 \pm 0.04$ ( $\mathrm{nmol} / \mathrm{mg}$ protein), which were significantly different from the control group.

\section{Discussion}

In cancer therapy, the combination therapeutic regimen regulating autophagic pathway has important therapeutic effects (26). Mechanistic target of rapamycin (mTOR) is an 
essential mediator in tumorigenesis, and rapamycin has been shown to induce cancer cell death by stimulating autophagy or apoptosis (9). Autophagy inhibition has been examined by many research laboratories, indicating that it may be a novel way of increasing the efficacy of anticancer agents (27).

Garlic has been used in traditional Chinese medicine (TCM) for centuries for its anti-inflammatory, cardiovascular protection, and anticancer effects (28). SAMC, a water-soluble garlic derivative, has been shown to have antiproliferation ability in many cancer cell lines, and tumor suppressing effect under in vivo conditions (29). Thus, we explored the anticancer effects of SAMC and combination use with rapamycin as well as their molecular mechanisms.

In this study, we showed that rapamycin suppressed tumor growth in the HCT-116 xenograft mouse model, and this effect was enhanced when combined with SAMC. In brief, the SAMC treatment upregulated the expression level of Bax, whereas rapamycin downregulated $\mathrm{Bcl}-2$. As a consequence, the combination of rapamycin and SAMC markedly raised the $\mathrm{Bax} / \mathrm{Bcl}-2$ ratio and significantly increased the induction of apoptosis in colorectal cancer. The p53-dependent apoptosis observed was in agreement with published results that the activation of p53 mediates the upregulation of Bax and downregulation of $\mathrm{Bcl}-2$ (30).

In cancer cells and its microenvironment, hypoxia and nutrient-deprivation condition could induce the autophagy process. Autophagy breaks down cellular damaged organelles and accumulates proteins for recycling, and the catabolites are recycled and used for biosynthesis and energy-metabolism as a cytoprotective response, which is essential for cancer cell survival (31). SAMC has been demonstrated to enhance autophagy in a liver disease model (32). We tested the autophagy activity in colorectal cancer tumor tissues. Western blotting results show that rapamycin and SAMC treatments increased the autophagic marker LC3-II protein expression. These results demonstrate that co-treatment of rapamycin and SAMC in colorectal cancer tumors can activate the autophagy pathway.

Because mTOR signaling contributes to drug resistance in patients (33), and one suggestion is that there is a mTORmediated negative feedback loop to Akt (34), we tested the Akt phosphorylation in colorectal cancer. The phosphorylation of Akt (p-Akt) expression was elevated in the rapamycin treatment group, which was reversed with the combination treatment. These results demonstrate that SAMC could be a good adjuvant with rapamycin in cancer treatment.

The hypoxia condition in tumors increases oxidative stress, which will activate the master regulator of antioxidant defense regulator Nrf2 which participates in tumor growth $(35,36)$. Garlic and DATS has been shown to participate in Nrf2antioxidant responses (37). In our study, western blotting and q-PCR analyses both confirmed that rapamycin increased Nrf2 expression and the downstream gene NQO-1, the antioxidant protein expression was further enhanced with the combination treatment, indicating that rapamycin and SAMC could suppress tumor growth by the Nrf 2 signaling pathway. Since the interaction between Nrf2 and autophagy plays a key role in tumorigenesis (38), we explored the autophagosome cargo protein p62/SQSTM1 expression. Previous study has demonstrated that autophagy can suppress tumorigenesis through elimination of p62. Our results indicated that p62 in tumor was downregulated by the rapamycin treatment with/without SAMC, which was consistent with the recent report that Nrf2Keap1 binding competed p62 (39) for autophagy degradation (40). So, the Nrf2-Keap1 system activated by rapamycin and SAMC co-treatment in HCT-116 xenograft mice could be related to autophagy through p62, which is a key pathway in tumorigenesis and cancer therapy.

It is well-known that levels of SOD, GSH-Px, CAT and MDA are considered as common indexes of tissue antioxidant status (41). The MDA level is widely used as a marker of lipid peroxidation damage. Furthermore, inhibition of antioxidant SOD, MDA and GSH enzymes is implicated in the pathogenesis conditions. SAMC has been reported to reduce oxidative stress and inflammation in liver-injury mice (42). Our study results show that rapamycin with/without SAMC treatment effectively increased the liver antioxidative capacity and alleviated the oxidative stress conditions.

In conclusion, these results showed that the rapamycin and SAMC combination induced apoptosis, activation of autophagy, and downregulation of p62. Additionally, this combination reversed the oxidative stress condition by activating antioxidative transcriptor $\mathrm{Nrf} 2$ and downstream gene NQO1 as well as increased SOD, GSH-Px, and CAT activities and decreased the MDA level in liver tissues. Therefore, combining rapamycin and SAMC for the treatment of colorectal cancer might be feasible in clinical use. The underlying mechanism of autophagy/p62/Nrf2 pathway revealed in this study may provide a new direction for drug development.

\section{Acknowledgements}

This study was supported by the funds from National 'Major Science and Technology Project-Prevention and Treatment of AIDS, Viral Hepatitis, and Other Major Infectious Diseases' (grant no. 2013ZX10005004), Major Project of Science and Technology of Shandong Province (grant no. 2015ZDJS04001), Science and Technology Enterprise Technology Innovation Fund of Jiangsu Province (grant no. BC2014172), Small and Medium Enterprise Technology Innovation Project of Lianyungang City (grant no. CK1333).

\section{References}

1. McGuire S: World Cancer Report 2014. Geneva, Switzerland: World Health Organization, International Agency for Research on Cancer, WHO Press, 2015. Adv Nutr 7: 418-419, 2016.

2. Ait Ouakrim D, Pizot C, Boniol M, Malvezzi M, Boniol M, Negri E, Bota M, Jenkins MA, Bleiberg H and Autier P: Trends in colorectal cancer mortality in Europe: Retrospective analysis of the WHO mortality database. BMJ 351: h4970, 2015.

3. White E: The role for autophagy in cancer. J Clin Invest 125 : 42-46, 2015.

4. White E: Deconvoluting the context-dependent role for autophagy in cancer. Nat Rev Cancer 12: 401-410, 2012.

5. Hu YL, Jahangiri A, Delay M and Aghi MK: Tumor cell autophagy as an adaptive response mediating resistance to treatments such as antiangiogenic therapy. Cancer Res 72: 4294-4299, 2012.

6. Kim YC and Guan KL: mTOR: A pharmacologic target for autophagy regulation. J Clin Invest 125: 25-32, 2015.

7. Xu K, Liu P and Wei W: mTOR signaling in tumorigenesis. Biochim Biophys Acta 1846: 638-654, 2014.

8. Mita MM, Mita A and Rowinsky EK: The molecular target of rapamycin (mTOR) as a therapeutic target against cancer. Cancer Biol Ther 2 (Suppl 1): S169-S177, 2003. 
9. Abraham RT and Gibbons JJ: The mammalian target of rapamycin signaling pathway: Twists and turns in the road to cancer therapy. Clin Cancer Res 13: 3109-3114, 2007.

10. Sun SY, Rosenberg LM, Wang X, Zhou Z, Yue P, Fu H and Khuri FR: Activation of Akt and eIF4E survival pathways by rapamycin-mediated mammalian target of rapamycin inhibition. Cancer Res 65: 7052-7058, 2005.

11. Rahman K and Lowe GM: Garlic and cardiovascular disease: A critical review. J Nutr 136 (Suppl): 736S-740S, 2006.

12. Lee Y: Induction of apoptosis by S-allylmercapto-L-cysteine, a biotransformed garlic derivative, on a human gastric cancer cell line. Int J Mol Med 21: 765-770, 2008.

13. Hu H, Zhang XP, Wang YL, Chua CW, Luk SU, Wong YC, Ling MT, Wang XF and Xu KX: Identification of a novel function of Id-1 in mediating the anticancer responses of SAMC, a watersoluble garlic derivative, in human bladder cancer cells. Mol Med Rep 4: 9-16, 2011.

14. Howard EW, Ling MT, Chua CW, Cheung HW, Wang X and Wong YC: Garlic-derived S-allylmercaptocysteine is a novel in vivo antimetastatic agent for androgen-independent prostate cancer. Clin Cancer Res 13: 1847-1856, 2007.

15. Lee Y, Kim H, Lee J and Kim K: Anticancer activity of S-allylmercapto-L-cysteine on implanted tumor of human gastric cancer cell. Biol Pharm Bull 34: 677-681, 2011.

16. Shirin H, Pinto JT, Kawabata Y, Soh JW, Delohery T, Moss SF, Murty V, Rivlin RS, Holt PR and Weinstein IB: Antiproliferative effects of S-allylmercaptocysteine on colon cancer cells when tested alone or in combination with sulindac sulfide. Cancer Res 61: 725-731, 2001

17. Zhang L, Li J, Ma J, Chen X, Chen K, Jiang Z, Zong L, Yu S, $\mathrm{Li}$ X, Xu Q, et al: The relevance of Nrf2 pathway and autophagy in pancreatic cancer cells upon stimulation of reactive oxygen species. Oxid Med Cell Longev 2016: 3897250, 2016.

18. Hiramatsu K, Tsuneyoshi T, Ogawa T and Morihara N: Aged garlic extract enhances heme oxygenase-1 and glutamatecysteine ligase modifier subunit expression via the nuclear factor erythroid 2-related factor 2-antioxidant response element signaling pathway in human endothelial cells. Nutr Res 36: 143-149, 2016

19. Xu XH, Li GL, Wang BA, Qin Y, Bai SR, Rong J, Deng T and Li Q: Diallyl trisufide protects against oxygen glucose deprivation -induced apoptosis by scavenging free radicals via the $\mathrm{PI} 3 \mathrm{~K} / \mathrm{Akt}$-mediated Nrf2/HO-1 signaling pathway in B35 neural cells. Brain Res 1614: 38-50, 2015.

20. Menegon S, Columbano A and Giordano S: The dual roles of NRF2 in cancer. Trends Mol Med 22: 578-593, 2016.

21. Starkenmann C, Niclass Y and Troccaz M: Nonvolatile S-alk(en) ylthio-L-cysteine derivatives in fresh onion (Allium cepa $\mathrm{L}$. cultivar). J Agric Food Chem 59: 9457-9465, 2011.

22. Zhang Y, Li HY, Zhang ZH, Bian HL and Lin G: Garlic-derived compound S-allylmercaptocysteine inhibits cell growth and induces apoptosis via the JNK and p38 pathways in human colorectal carcinoma cells. Oncol Lett 8: 2591-2596, 2014.

23. Tanida I, Minematsu-Ikeguchi N, Ueno T and Kominami E: Lysosomal turnover, but not a cellular level, of endogenous LC3 is a marker for autophagy. Autophagy 1: 84-91, 2005.

24. Willems L, Tamburini J, Chapuis N, Lacombe C, Mayeux P and Bouscary D: PI3K and mTOR signaling pathways in cancer: New data on targeted therapies. Curr Oncol Rep 14: 129-138, 2012.

25. Mathew R, Karp CM, Beaudoin B, Vuong N, Chen G, Chen HY, Bray K, Reddy A, Bhanot G, Gelinas C, et al: Autophagy suppresses tumorigenesis through elimination of p62. Cell 137: $1062-1075,2009$.

26. Alayev A, Berger SM, Kramer MY, Schwartz NS and Holz MK: The combination of rapamycin and resveratrol blocks autophagy and induces apoptosis in breast cancer cells. J Cell Biochem 116: 450-457, 2015
27. Amaravadi RK, Lippincott-Schwartz J, Yin XM, Weiss WA, Takebe N, Timmer W, DiPaola RS, Lotze MT and White E: Principles and current strategies for targeting autophagy for cancer treatment. Clin Cancer Res 17: 654-666, 2011.

28. Thomson M and Ali M: Garlic [Allium sativum]: A review of its potential use as an anticancer agent. Curr Cancer Drug Targets 3: 67-81, 2003.

29. Liang D, Qin Y, Zhao W, Zhai X, Guo Z, Wang R, Tong L, Lin L, Chen $\mathrm{H}$, Wong YC, et al: S-allylmercaptocysteine effectively inhibits the proliferation of colorectal cancer cells under in vitro and in vivo conditions. Cancer Lett 310: 69-76, 2011.

30. Wang XW: Role of p53 and apoptosis in carcinogenesis. Anticancer Res 19A: 4759-4771, 1999.

31. Viry E, Paggetti J, Baginska J, Mgrditchian T, Berchem G, Moussay E and Janji B: Autophagy: An adaptive metabolic response to stress shaping the antitumor immunity. Biochem Pharmacol 92: 31-42, 2014.

32. Xiao J, Guo R, Fung ML, Liong EC, Chang RC, Ching YP and Tipoe GL: Garlic-derived S-Allylmercaptocysteine ameliorates nonalcoholic fatty liver dsease in a rat model through inhibition of apoptosis and enhancing autophagy. Evid Based Complement Alternat Med 2013: 642920, 2013.

33. Jiang BH and Liu LZ: Role of mTOR in anticancer drug resistance: Perspectives for improved drug treatment. Drug Resist Updat 11: 63-76, 2008.

34. Feldman ME and Shokat KM: New inhibitors of the PI3KAkt-mTOR pathway: Insights into mTOR signaling from a new generation of Tor Kinase Domain Inhibitors (TORKinibs). Curr Top Microbiol Immunol 347: 241-262, 2010.

35. Leinonen HM, Kansanen E, Pölönen P, Heinäniemi $M$ and Levonen AL: Role of the Keap1-Nrf2 pathway in cancer. Adv Cancer Res 122: 281-320, 2014.

36. Moon EJ and Giaccia A: Dual roles of NRF2 in tumor prevention and progression: Possible implications in cancer treatment. Free Radic Biol Med 79: 292-299, 2015.

37. Padiya R, Chowdhury D, Borkar R, Srinivas R, Pal Bhadra M and Banerjee SK: Garlic attenuates cardiac oxidative stress via activation of PI3K/AKT/Nrf2-Keap1 pathway in fructose-fed diabetic rat. PLoS One 9: e94228, 2014

38. Chen N, Wu L, Yuan H and Wang J: ROS/Autophagy/Nrf2 pathway mediated low-dose radiation induced radio-resistance in human lung adenocarcinoma A549 Cell. Int J Biol Sci 11: $833-844,2015$

39. Komatsu M, Kurokawa H, Waguri S, Taguchi K, Kobayashi A, Ichimura Y, Sou YS, Ueno I, Sakamoto A, Tong KI, et al: The selective autophagy substrate p62 activates the stress responsive transcription factor Nrf2 through inactivation of Keap1. Nat Cell Biol 12: 213-223, 2010.

40. Jiang T, Harder B, Rojo de la Vega M, Wong PK, Chapman E and Zhang DD: p62 links autophagy and Nrf2 signaling. Free Radic Biol Med 88B: 199-204, 2015.

41. Cigremis Y, Turel H, Adiguzel K, Akgoz M, Kart A, Karaman M and Ozen $\mathrm{H}$ : The effects of acute acetaminophen toxicity on hepatic mRNA expression of SOD, CAT, GSH-Px, and levels of peroxynitrite, nitric oxide, reduced glutathione, and malondialdehyde in rabbit. Mol Cell Biochem 323: 31-38, 2009.

42. Xiao J, Liong EC, Ling MT, Ching YP, Fung ML and Tipoe GL: S-allylmercaptocysteine reduces carbon tetrachloride-induced hepatic oxidative stress and necroinflammation via nuclear factor kappa B-dependent pathways in mice. Eur J Nutr 51: 323-333, 2012. 